\title{
A GEOMETRICAL CHARACTERIZATION OF BANACH SPACES WITH THE RADON-NIKODYM PROPERTY
}

BY

\author{
HUGH B. MAYNARD
}

\begin{abstract}
A characterization of Banach spaces having the Radon-Nikodym property is obtained in terms of a convexity requirement on all bounded subsets. In addition a Radon-Nikodym theorem, utilizing this convexity property, is given for the Bochner integral and it is easily shown that this theorem is equivalent to the Phillips-Metivier Radon-Nikodym theorem as well as all the standard Radon-Nikodym theorems for the Bochner integral.
\end{abstract}

1. Introduction. Rieffel [9] proved a Radon-Nikodym theorem for the Bochner integral, using techniques established in [8], in an attempt to establish the RadonNikodym theorem of Phillips [7] and Metivier [5]. He was unable to establish it in the nonseparable case, the result depending upon a proof that every convex weakly compact set in a $B$-space is dentable. This circle of ideas was not closed until Troyanski [10] proved that a Banach space with a weakly compact fundamental subset is isomorphic to a locally uniformly convex Banach space. This is, as would be expected, much deeper than necessary and a simpler proof will be indicated in $\$ 2$.

The obvious characterization of Banach spaces with the Radon-Nikodym property would seem to be that every bounded subset must be dentable. In $\$ 3$ it is demonstrated that a characterization is that every bounded subset must be $\sigma$ dentable, where $\sigma$-dentability is a dentable type condition which is strictly weaker than dentability. It is however an open question if dentable and o-dentable coincide in Banach spaces having the Radon-Nikodym property.

2. Dentability and $\sigma$-dentability with application to Phillip's Radon-Nikodym theorem. The following notation will be observed in the remainder of this paper. $B$ will denote a Banach space and if $D \subset B$ then $c(D)$ and $c(D)$ will denote the convex hull of $D$ and the closed convex hull of $D$, respectively. The open and closed spheres of radius $r$ about $x \in B$ will be $S_{r}(x)$ and $\bar{S}_{r}(x)$. If $(X, \Sigma, \mu)$ is a totally finite positive measure space then $\Sigma^{+}=\{E \in \Sigma: \mu(E)>0\}$ and for a $B$ valued measure $m$ on $\Sigma$, the average range of $m$ over $E \in \Sigma^{+}$with respect to $\mu$ is $A_{E}(m)=\left\{m(F) / \mu(F): F \subset E, F \in \Sigma^{+}\right\}$.

Definition 2.1. A set $D \subset B$ is o-convex iff for every sequence $\left\{a_{i}\right\}_{i=1}^{\infty}, a_{i} \geq 0$, $\sum_{i=1}^{\infty} a_{i}=1$, and for every sequence $\left\{d_{i}\right\}_{i=1}^{\infty} \subset D$ such that $\sum_{i=1}^{\infty} a_{i} d_{i}$ converges, we have $\sum_{i=1}^{\infty} a_{i} d_{i} \in D$.

Received by the editors November 29, 1971 and, in revised form, December 5, 1972.

AMS (MOS) subject classifications (1970). Primary 28A45, 46B99, 46G10.

Key words and phrases. Radon-Nikodym theorem, Radon-Nikodym property, Bochner integral, dentable, $\sigma$-dentable. 
The o-convex hull of $D \subset B$ is given by

$$
\sigma(D)=\left\{\sum_{i=1}^{\infty} a_{i} d_{i}: a_{i} \geq 0, \sum_{i=1}^{\infty} a_{i}=1, \text { and } \sum_{i=1}^{\infty} a_{i} d_{i} \text { converges }\right\} .
$$

If $D$ is bounded then the infinite convex sums in $\sigma(D)$ always exist. We may also assume that the constants $a_{i}>0$. In addition we always have the following relations:

$$
D \subset c(D) \subset \sigma(D) \subset \bar{c}(D)
$$

where the inclusions may be strict.

We now recall the definition of dentable and introduce the concept of $\sigma$ dentable.

Definition 2.2. A set $D \subset B$ is dentable [0-dentable] iff for each $\varepsilon>0$ there exists $d \in D$, such that

$$
d \notin \bar{c}\left(D \sim S_{\varepsilon}(d)\right) \quad\left[d \notin \sigma\left(D \sim S_{\varepsilon}(d)\right)\right] .
$$

If $D$ is not dentable [ $\sigma$-dentable] then any number $\varepsilon>0$ such that for all $d \in D, d \in \bar{c}\left(D \sim S_{\varepsilon}(d)\right)\left[d \in \sigma\left(D \sim S_{\varepsilon}(d)\right)\right]$ is called a dentable limit [odentable limit] for the set $D$.

The following lemma is immediate.

Lemma 2.1. If $D \subset B$ is dentable then it is $\sigma$-dentable.

Example. By considering the following subset of $L^{\prime}(X, \Sigma, \mu)$ where $(X, \Sigma, \mu)$ is a nonatomic, finite, positive measure space with $\mu(X)=1$, we can see that $\sigma$ dentable is a strictly weaker concept than dentable.

Let $P$ be the positive cone in $L^{1}(X, \Sigma, \mu)$ and $U_{1}$ be the unit cell $\left[U_{1}\right.$ $=\{f:\|f\|=1\}]$ in $L^{1}(X, \Sigma, \mu)$. Then if $D=\left[\cup_{0<\theta<\pi} e^{i \theta} P \cap U_{1}\right] \cup\{1\}$ it is easy to establish that the constant function 1 is a $\sigma$-denting point for $D$ [i.e. $\forall \varepsilon>0$, 1 is the appropriate element of $D]$ and yet $D$ is not dentable.

In order to prove dentability or $\sigma$-dentability of a set it is often possible to reduce the problem to the consideration of countable sets.

Lemma 2.2. If $D \subset B$ has the property that every countable subset is dentable ( $\sigma$ dentable) then $D$ is dentable ( $\sigma$-dentable).

Proof. The proof of the $\sigma$-dentable assertion is entirely analogous to that of the dentable case and thus we will only prove the dentable assertion.

Suppose $D$ is not dentable. Then there exists $\varepsilon>0$ such that $\varepsilon$ is a dentable limit for $D$. Now for each $x \in D$ there exists a countable set $A_{x} \subset D \sim S_{e}(x)$ such that $x \in \bar{c}\left(A_{x}\right)$.

Define by induction a sequence $\left\{A_{n}\right\}$ of subsets as follows. Pick any $z \in D$ and set $A_{1}=\{z\}$. Given $A_{n-1}$ let $A_{n}=\bigcup\left\{A_{x}: x \in A_{n-1}\right\}$. Thus the set $A=\bigcup_{n=1}^{\infty} A_{n}$ $\subset D$ is countable and is clearly not dentable and hence the lemma is established. 
Theorem 2.1. If $K \subset B$ is a relatively weakly compact set, then it is dentable.

Proof. By Lemma 2.2 we need only consider the case when $B$ is separable. In this case the argument given by Rieffel $[9$, p. 76$]$ or the argument by Namioka $[6$, p. 150] can be used to obtain the result.

An elementary proof of this fact can be obtained in the following manner.

It suffices to assume that $K$ is a convex weakly compact set since Rieffel [9] showed that if $\bar{c}(D)$ is dentable then $D$ is dentable.

Then by Lemma 2.2 it suffices to assume that $B$ is separable. Suppose $\varepsilon>0$ and let $A$ be the set of extreme points of $K$. By the Krein-Milman theorem, $A \neq \varnothing$. Let $\left\{x_{i}\right\}_{i=1}^{\infty}$ be a dense subset in $B$; then since $\bar{A}^{w}$ is weakly compact and since

$$
\bar{A}^{w}=\bigcup_{i=1}^{\infty} \bar{A}^{w} \cap\left[x_{i}+\bar{S}_{\mathrm{e} / 2}(0)\right]
$$

there exists at least one $i$ and a weak convex neighborhood $N$ such that $\bar{A}^{w} \cap\left[x_{i}+\bar{S}_{\ell / 2}(0)\right]$ contains $N \cap \bar{A}^{w}$. This follows since $\bar{A}^{w}$ is a Baire space and since $\bar{S}_{\varepsilon / 2}(0)=\bar{S}_{\varepsilon / 2}^{w}(0)$.

Thus there exists $x \in A$ such that $x$ is in the interior of $N$ and the diameter of $N \cap \bar{A}^{w}$ is bounded by $\varepsilon / 2$.

Let $K_{1}=\bar{c}(K \sim N), K_{2}=\bar{c}(N \cap A) . K_{1}$ and $K_{2}$ are both weakly compact, convex, and disjoint. Thus

$$
\begin{aligned}
c\left(K_{1}\right. & \left.\cup K_{2}\right)=\bar{c}\left(K_{1} \cup K_{2}\right) \\
& =\left\{\lambda x_{1}+(1-\lambda) x_{2}: 0 \leq \lambda \leq 1, x_{1} \in K_{1}, x_{2} \in K_{2}\right\} .
\end{aligned}
$$

The diameters of $K_{1}$ and $K_{2}$ have the following bounds: $\delta\left(K_{2}\right) \leq \varepsilon / 2$ and if $d=\delta(K)<\infty, \delta\left(K_{i}\right) \leq d$. Assume $d \neq 0$. Let $C=\left\{\lambda x_{1}+(1-\lambda) x_{2}: x_{1}\right.$ $\left.\in K_{1}, x_{2} \in K_{2}, \varepsilon / 4 d \leq \lambda \leq 1\right\}$. Thus $C \supset K_{1}$ and $C$ is weakly compact. Suppose $y_{1}, y_{2} \in K \sim C$. Then

$$
y_{\iota}=\lambda_{\imath} x_{1}^{\imath}+\left(1-\lambda_{\imath}\right) x_{2}^{\iota}, \quad 0 \leq \lambda_{\imath}<\varepsilon / 4 d, x_{1}^{\imath} \in K_{1}, x_{2}^{\iota} \in K_{2}, \iota=1,2 .
$$

Thus

$$
\begin{aligned}
\left\|y_{1}-y_{2}\right\| & \leq\left|\lambda_{1}\right|\left\|x_{1}^{1}-x_{2}^{1}\right\|+\left\|x_{2}^{1}-x_{2}^{2}\right\|+\left|\lambda_{2}\right|\left\|x_{1}^{2}-x_{2}^{2}\right\| \\
& <(\varepsilon / 4 d) \cdot d+\varepsilon / 2+(\varepsilon / 4 d) \cdot d=\varepsilon .
\end{aligned}
$$

Thus if $N_{1}=N \sim C, N_{1}$ is weakly open, $x \in N_{1}$, and the diameter of $N_{1} \cap K$ is less than $\varepsilon$. Thus $x \notin K \sim S_{\varepsilon}(x)$ since $S_{\varepsilon}(x) \supset N_{1} \cap K$. Thus since $x$ is an extreme point of $K, x \notin \bar{c}\left(K \sim S_{\varepsilon}(x)\right)$ and $K$ is dentable.

The following theorem is due to Rieffel [9, Theorem 1, p. 71] and is obtained by replacing dentable with $\sigma$-dentable, the proof remaining essentially the same. We include a proof using the locally small average range Radon-Nikodym 
theorem [4, Theorem 3.1] in the spirit of the simple equivalence of all RadonNikodym theorems for the Bochner integral.

Theorem 2.2. Let $(X, \Sigma, \mu)$ be a totally finite positive measure space and let $B$ be a Banach space. Let $m$ be a B-valued measure on $\Sigma$. Then there is a B-valued Bochner integrable function $f$ on $X$ such that $m(E)=\int_{E} f d \mu$ for all $E \in \Sigma$, iff

(i) $m$ is $\mu$-continuous,

(ii) $|m|(X)<\infty$,

(iii) $m$ has locally $\sigma$-dentable average range, that is, given $E \in \Sigma^{+}$, there exists $F \subset E, F \in \Sigma^{+}$, such that $A_{F}(m)$ is $\sigma$-dentable.

Proof. $(\Rightarrow)$ This is immediate from Theorem $1, \operatorname{Rieffel}[9$, p. 71$]$ and Lemma 2.1.

$(\Leftrightarrow)$ Let $E \in \Sigma^{+}$and $\varepsilon>0$ be given. Then there exists $E_{d} \subset E, E_{d} \in \Sigma^{+}$, such that $A_{E_{d}}(m)$ is $\sigma$-dentable. Thus choose $b \in A_{E_{d}}(m)$ such that $b \notin$ $\sigma\left(A_{E_{d}}(m)-S_{e}(b)\right)$. Suppose $b=m\left(F_{0}\right) / \mu\left(F_{0}\right), F_{0} \subset E_{d}, F_{0} \in \Sigma^{+}$. Then by Theorem 3.1 and its corollary [4, p. 16], if $b \in A\left(F_{0}, \varepsilon\right)=\{r \in B:\|m(A)-r \mu(A)\|$ $\left.\leq \varepsilon \mu(A), \forall A \subset F_{0}, A \in \Sigma^{+}\right\}$we are done. So suppose $b \notin A\left(F_{0}, \varepsilon\right)$.

Claim. There exists $F \subset F_{0}, F \in \Sigma^{+}$, such that $b \in A(F, \varepsilon)$.

Proof. Suppose not. Then the property that $\|m(\tilde{E}) / \mu(\tilde{E})-b\|>\varepsilon$ is a local null difference property and hence by the exhaustion principle [4, Lemma 1.1, p. 2] $F_{0}=\cup_{i=1}^{\infty} E_{i}$ where $m\left(E_{i}\right) / \mu\left(E_{i}\right) \in A_{F_{0}}(m) \sim S_{e}(b) \subset A_{E_{d}}(m) \sim S_{\varepsilon}(b)$, but $m\left(F_{0}\right) / \mu\left(F_{0}\right)=\sum_{i=1}^{\infty}\left(\mu\left(E_{i}\right) / \mu\left(F_{0}\right)\right) m\left(E_{i}\right) / \mu\left(E_{i}\right) \in \sigma\left(A_{E_{d}}(m) \sim S_{e}(b)\right)$ and this yields a contradiction.

Thus there must exist $F \subset F_{0} \subset E, F \in \Sigma^{+}$, such that $b \in A(F, \varepsilon)$ and by Theorem 3.1 and its corollary $[4$, p. 16] we have the desired conclusion.

Corollary [Phillips]. Let $(X, \Sigma, \mu)$ be a totally finite positive measure space and let $B$ be a Banach space. Let $m$ be a B-valued measure on $\Sigma$. Then there is a B-valued Bochner integrable function $f$ on $X$, such that $m(E)=\int_{E} f d \mu$, for all $E \in \Sigma$, iff

(i) $m$ is $\mu$-continuous,

(ii) $|m|(X)<\infty$, and

(iii) $m$ has locally relatively weakly compact average range.

Proof. $(\Rightarrow)$ This follows from Rieffel $[8$, p. 466].

$(\Leftarrow)$ If $m$ has locally relatively weakly compact average range then, by Theorem 2.1, $m$ has locally dentable average range.

3. A geometric characterization of Banach spaces with the Radon-Nikodym property. The concept of $\sigma$-dentability allows us to obtain a relatively simple characterization of Banach spaces with the Radon-Nikodym property using Theorem 2.2.

Definition. A Banach space $B$ has the Radon-Nikodym property (R-N property) iff for any totally finite positive measure space $(X, \Sigma, \mu)$ and any $B$ - 
valued $\mu$-continuous measure $m$ on $\Sigma$, with $|m|(X)<\infty$, there exists $f \in$ $L_{B}^{\prime}(X, \Sigma, \mu)$ such that $m(E)=\int_{E} f d \mu$ for all $E \in \Sigma$.

Definition. A Banach space $B$ is said to be a $\sigma$-dentable space iff every bounded set $K \subset B$ is $\sigma$-dentable.

It should be emphasized that it is not known if a $\sigma$-dentable space need have all of its bounded subsets dentable.

Theorem 3.1. $A$ Banach space $B$ has the Radon-Nikodym property iff $B$ is $a \sigma$ dentable space.

Proof. $(\Leftarrow)$ If $B$ is a $\sigma$-dentable space then Theorem 2.2 immediately implies that $B$ has the R-N property because any $B$-valued, $\mu$-continuous measure of finite variation has locally bounded average range.

$(\Rightarrow)$ Suppose $B$ is not a $\sigma$-dentable space. Then there exists a bounded subset $K \subset B$ such that $K$ is not $\sigma$-dentable. We will construct two regular measures $m$ and $\mu$ which negate the Radon-Nikodym property.

Since $K$ is bounded and not $\sigma$-dentable we can choose $\varepsilon, N$ such that

(i) $\varepsilon$ is a $\sigma$-dentable limit for $K$, and

(ii) $K \subset S_{N}(0)$.

Let $X=[0,1)$ and choose an increasing sequence of infinite partitions $\left\{\pi_{n}\right\}_{n=1}^{\infty}$ of $X$ such that the following conditions are satisfied:

(i) $\pi_{n}=\left\{A_{z}^{n}\right\}_{z \in N^{n}}$ where each $A_{z}^{n}=\left[a_{z}^{n}, b_{z}^{n}\right)$.

(ii) For each $n, z \in N^{n}, A_{z}^{n}=\bigcup_{i=1}^{\infty} A_{(z, i)}^{n+1}$ where we consider $(z, i) \in N^{n+1}$.

(iii) For each $n, z \in N^{n}, b_{(2, i)}^{n+1}=a_{(2, i+1)}^{n+1}$. Thus the decomposition of each half open interval $A_{z}^{n}$ proceeds from left to right.

We now define a ring of subsets $\subset$ of $X$. Let $\mathcal{Q}=\{A \cup B: A$ is a finite union of $A_{2}^{k}$ 's and $B$ is a finite union of sets of the form $\left.\cup_{i=m}^{\infty} A_{(2, i)}^{n+1}=A_{z}^{n} \sim \cup_{i=1}^{m-1} A_{(2, i)}^{n+1}\right\}$.

We consider both $\varnothing$ and $X$ to be elements of $\delta$. We will now define $\mu$ and $m$ on $\mathcal{L}$ and extend to regular countably additive measures on $\sigma(\alpha P)$, the $\sigma$-algebra generated by $\subset$. $\sigma(\subset R)$ consists of the Burel subsets of $[0,1)$.

Define $\mu$ and $m$ by induction on the sequence of partitions. Let $\mu(\varnothing)=0$, $m(\varnothing)=0, \mu(X)=1, m(X)=k$ where $k$ is any element of $K$. Suppose $\mu$ and $m$ are defined on the elements of $\pi_{n}$ such that $m\left(A_{2}^{n}\right) / \mu\left(A_{z}^{n}\right)=k_{z}^{n} \in K$ for each $A_{z}^{n} \in \pi_{n}$. Then since $K$ is not $\sigma$-dentable, $k_{z}^{n}=\sum_{i=1}^{\infty} \alpha_{(2, i)}^{n+1} k_{(2, i)}^{n+1}, \alpha_{(2, i)}^{n+1}>0$, $\sum_{i=1}^{\infty} \alpha_{(2, i)}^{n+1}=1$ and $\left\{k_{(2, i)}^{n+1}\right\}_{i=1}^{\infty} \subset K \sim S_{e}\left(k_{z}^{n}\right)$. We now define $\mu\left(A_{(z, i)}^{n+1}\right)$ $=\alpha_{(2, i)}^{n+1} \mu\left(A_{z}^{n}\right)$ and $m\left(A_{(z, i)}^{n+1}\right)=\mu\left(A_{(z, i)}^{n+1}\right) k_{(z, i)}^{n+1}$. Let $\pi=\left\{A \subset X: A \in \pi_{n}\right.$ for some n).

Thus $m$ and $\mu$ are defined on each $\pi_{n}$ and hence can be extended by finite additivity to all of $C R$.

Notice that the diameter of the average range of $m$ over each $A_{z}^{n}$ is at least $\varepsilon$. This fact, after extension to $\sigma(\sigma P)$, will yield the contradiction.

Notice also that the construction yields a "horizontal" countable additivity, that is, 


$$
\begin{array}{ll}
\mu\left(A_{z}^{n}\right)=\sum_{i=1}^{\infty} \mu\left(A_{(z, i)}^{n+1}\right) & \text { and } \\
m\left(A_{z}^{n}\right)=\sum_{i=1}^{\infty} m\left(A_{(z, i)}^{n+1}\right) & \text { for all } n \in N, z \in N^{n} .
\end{array}
$$

Claim 1. $\mu$ can be extended to a Borel measure on $[0,1)$ and hence is regular and countably additive.

Proof. It suffices to show that $\mu$ is regular on $\pi$ relative to $\mathcal{R}$ since it is then regular on $\mathcal{R}$ and hence has an extension to a Borel measure on $[0,1)$.

Let $\varepsilon>0$ be arbitrary and $A \in \pi$. Then using the "horizontal" countable additivity there exists $\left\{A_{i}\right\}_{i=1}^{n} \subset \pi$ such that

$$
\left|\mu(A)-\sum_{i=1}^{m} \mu\left(A_{i}\right)\right|<\varepsilon .
$$

Thus we have

$$
\bigcup_{i=1}^{m} A_{i} \subset \overline{\bigcup_{i=1}^{m} A_{i}} \subset A,
$$

$\overline{\bigcup_{i=1}^{m} A_{i}}$ is compact and hence $\mu$ is inner regular on $A$.

Suppose $A=[a, b)$. Then by choosing the tail end of the decomposition of the preceding interval, we can find a sequence $\left\{A_{i}\right\}_{i=1}^{\infty}$ such that $\mu\left(\cup_{i=1}^{\infty} A_{i}\right)<\varepsilon$, $\cup_{i=1}^{\infty} A_{i} \in \mathcal{R}$, and $\left(A \cup\left[\cup_{i=1}^{\infty} A_{i}\right]\right)^{\iota} \supset A$, where $D^{\iota}$ is the interior of $D$. Thus $\mu$ is outer regular on $A$ and hence $\mu$ is regular on all of $\pi$.

Claim 2. $m$ can be extended to a Borel measure on $[0,1)$ such that $\|m(A)\|$ $\leq N \mu(A)$ for all $A \in \sigma(\propto P)$. Thus the extension is countably additive and regular.

Proof. Since $\mu$ is regular and dominates $m$ we can apply Theorem $1[1$, p. 62] of Dinculeanu which implies that $m$ has a countably additive extension of finite variation such that $m$ remains dominated by $\mu$ and $m$ is regular.

Claim 3. $m$ is not an indefinite integral with respect to $\mu$.

Proof. It suffices to show that, for $B \in \sigma(\mathcal{L})$, the average range of $m$ over $B$, $A_{B}(m)$, has diameter not less that $\varepsilon / 2$. This sufficiency follows from Theorem 3.1 and its corollary $[4$, p. 16].

Let $B \in \sigma(\mathcal{L})$. Now by the regularity of $\mu$ and $m$ on $\sigma(\alpha R)$ we can choose a compact $C$ and an open $O$ such that (i) $C \subset B \subset O$, and (ii) $\mu(O-C)$ $<(\varepsilon / 16 N) \mu(B)$.

Now those elements in $\mathcal{R}$ of the form $A_{(2, i)}^{n} \cup\left[\cup_{i=m}^{\infty} A_{(2, i-1)}^{n}\right]$ form a base of the topology in $[0,1)$ and hence by the compactness of $C$ and the openness of $O$ we can find a finite number of these which cover $C$ and are contained in $O$. Thus there exists a disjoint sequence $\left\{A_{i}\right\}_{i=1}^{\infty} \subset \pi$ such that $C \subset \cup_{i=1}^{\infty} A_{i} \subset O$.

Now there must exist at least one set $A_{i}$ such that $\mu\left(A_{i} \sim B\right) / \mu\left(A_{i}\right)<\varepsilon / 8 N$ $=\delta$ since if not, we have 


$$
\begin{aligned}
\mu(O \sim C) & \geq \mu\left(\bigcup_{i=1}^{\infty} A_{i} \sim B\right)=\sum_{i=1}^{\infty} \mu\left(A_{i} \sim B\right) \\
& \geq \delta \sum_{i=1}^{\infty} \mu\left(A_{i}\right) \geq \delta \mu(C)\left[1-\frac{\varepsilon}{8 N}\right] \\
& \geq(\varepsilon / 16 N) \mu(B) \quad \Rightarrow \Leftarrow .
\end{aligned}
$$

Thus choose $A_{\alpha}$ such that

$$
\mu\left(A_{\alpha} \sim B\right) / \mu\left(A_{\alpha}\right)<\varepsilon / 8 N .
$$

Let $D=A_{\alpha} \cap B \in \sigma(\propto R)$, then $D \subset B$ and $\mu(D)>0$. Now by taking the next partition of $A_{\alpha}$ we get $A_{\alpha}=\cup_{k=1}^{\infty} C_{k}$ where the $\left\{C_{k}\right\}_{k=1}^{\infty} \subset \pi$ and are disjoint. Then there must exist a small $n$ such that

$$
\mu\left(C_{n} \sim B\right)<(\varepsilon / 8 N) \mu\left(C_{n}\right)
$$

since if not $\mu\left(A_{\alpha} \sim B\right)=\mu\left(\cup_{i=1}^{\infty}\left(C_{n} \sim B\right)\right) \geq(\varepsilon / 8 N) \sum_{i=1}^{\infty} \mu\left(C_{n}\right)$ $=(\varepsilon / 8 N) \mu\left(A_{\alpha}\right)$ which contradicts $(*)$.

Let $E=C_{n} \cap B$. Now from the construction of $m$ and $\mu$

$$
\left\|\frac{m\left(A_{\alpha}\right)}{\mu\left(A_{\alpha}\right)}-\frac{m\left(C_{n}\right)}{\mu\left(C_{n}\right)}\right\| \geq \varepsilon .
$$

In addition

$$
\begin{aligned}
\left\|\frac{m(D)}{\mu(D)}-\frac{m\left(A_{\alpha}\right)}{\mu\left(A_{\alpha}\right)}\right\| & =\left\|\left(1-\frac{\mu(D)}{\mu\left(A_{\alpha}\right)} \frac{m(D)}{\mu(D)}+\frac{\mu\left(A_{\alpha} \sim B\right)}{\mu\left(A_{\alpha}\right)} \frac{m\left(A_{\alpha} \sim B\right)}{\mu\left(A_{\alpha} \sim B\right)}\right)\right\| \\
& \leq \frac{\mu\left(A_{\alpha} \sim B\right)}{\mu\left(A_{\alpha}\right)}\left\{\left\|\frac{m(D)}{\mu(D)}\right\|+\left\|\frac{m\left(A_{\alpha} \sim B\right)}{\mu\left(A_{\alpha} \sim b\right)}\right\|\right\}<\frac{\varepsilon}{4} .
\end{aligned}
$$

In the same manner we get $\left\|m(E) / \mu(E)-m\left(C_{n}\right) / \mu\left(C_{n}\right)\right\|<\varepsilon / 4$. Thus

$$
\left\|\frac{m(E)}{\mu(E)}-\frac{m(D)}{\mu(D)}\right\| \geq \frac{\varepsilon}{2} .
$$

Thus the diameter of $A_{B}(m)$ is not less than $\varepsilon / 2$ for all $B \in \sigma(\alpha P)$ and hence $m$ is not an indefinite integral with respect to $\mu$.

Thus $B$ does not have the $\mathrm{R}-\mathrm{N}$ property.

The following corollary is due to Uhl [11, Theorem 1, p. 2].

Corollary. If $B$ is a Banach space such that every closed separable subspace of $B$ is linearly homeomorphic to a subspace of a separable dual space, then $B$ has the Radon-Nikodym property.

Proof. Suppose $B$ satisfies the hypothesis of the corollary. Let $K$ be any bounded set in $B$ and $D$ any countable subset of $K$. Then the closed linear span 
$[D]$ of $D$ is linearly homeomorphic to a subspace of a separable dual space. Since a linear homeomorphism maps $\sigma$-dentable sets into $\sigma$-dentable sets and since a separable dual space has the R-N property, $D$ is mapped into a $\sigma$-dentable set and hence is itself $\sigma$-dentable. Thus $K$ is $\sigma$-dentable and $B$ has the R-N property.

\section{REFERENCES}

1. N. Dinculeanu, Vector measures, Internat. Series of Monographs in Pure and Appl. Math., vol. 95, Pergamon Press, New York, 1967. MR 34 \#6011b.

2. James E. Honeycutt, Jr., Extensions of abstract valued set functions, Trans. Amer. Math. Soc. 141 (1969), 505-513.

3. J. Lindenstrauss, On operators which attain their norm, Israel J. Math. 1(1963), 139-148. MR 28 \#3308.

4. H. B. Maynard, A Radon-Nikodým theorem for operator-valued measures, Trans. Amer. Math. Soc. 173 (1972), 449-463.

5. M. Métivier, Martingales à valeurs vectorielles. Applications d la dérivation des mesures vectorielles, Ann. Inst. Fourier (Grenoble) 17(1967), 175-208. MR 40 \#926.

6. I. Namioka, Neighborhoods of extreme points, Israel J. Math. 5(1967), 145-152. MR 36 \#323.

7. R. S. Phillips, On weakly compact subsets of a Banach space, Amer. J. Math. 65(1943), 108-136. MR 4, 218.

8. M. A. Rieffel, The Radon-Nikodym theorem for the Bochner integral, Trans. Amer. Math. Soc. 131 (1968), 466-487. MR 36\#5297.

9. Dentable subsets of Banach spaces, with application to a Radon-Nikodym theorem, Proc. Conf. Functional Analysis (Irvine, Calif., 1966), Thompson Book, Washington, D.C., 1967, pp. 71-77. MR 36\#5668.

10. S. L. Troyanski, On locally uniformly convex and differentiable norms in certain non-separable Banach spaces, Studia Math. 38(1971), 173-180.

11. J. J. Uhl, Jr., A note on the Radon-Nikodym property for Banach spaces (preprint).

Department of Mathematics, University of Utah, Salt Lake City, Utah 84112 